\title{
Passive transfer of fibromyalgia pain from patients to mice.
}

Andreas Goebel ${ }^{1 \#}$, Clive Gentry², Ulku Cuhadar², Emerson Krock ${ }^{3}$, Nisha Vastani² ${ }^{2}$ Serena

Sensi ${ }^{1}$, Katalin Sandor ${ }^{3}$, Alexandra Jurczak ${ }^{3}$, Azar Baharpoor ${ }^{3}$, Louisa Brieskorn ${ }^{3}$, Carlos Morado Urbina ${ }^{3}$, Angelika Sandstrom ${ }^{4}$, Janette Tour ${ }^{4}$, Diana Kadetoff ${ }^{4,5}$, Eva Kosek ${ }^{4}$, Stuart Bevan $^{2 \#}$, Camilla I. Svensson ${ }^{3 *}$ and David A. Andersson ${ }^{2 * \#}$

*Authors contributed equally

"Corresponding Author

${ }^{1}$ Pain Research Institute, Department of Translational Medicine, University of Liverpool, UK; ${ }^{2}$ King's College London, Wolfson CARD, Institute of Psychiatry, Psychology \& Neuroscience, Guy's Campus, SE1 1UL; ${ }^{3}$ Department of Physiology and Pharmacology, Karolinska Institutet, 17177 Stockholm, Sweden; ${ }^{4}$ Department of Clinical Neuroscience, Karolinska Institutet, 17177 Stockholm, Sweden; ${ }^{5}$ Stockholm Spine Center, 19489 Upplands Väsby, Sweden. 


\section{SUMMARY}

Fibromyalgia syndrome (FMS) is a chronic pain condition characterized by widespread pain and tenderness ${ }^{1,2}$. The etiology and pathophysiology of fibromyalgia are unknown and there are no effective treatments. Here we show that sensory hypersensitivity in FMS is caused by autoantibodies that act by sensitizing nociceptive sensory neurons. Administration of IgG from FMS patients increased mouse pain sensitivities to stimulation with mechanical pressure and cold. In contrast, transfer of IgG depleted samples from FMS patients or IgG from healthy control subjects had no effect on pain sensitivity. Sensory nerve fibres in ex vivo skin-nerve preparations from mice treated with FMS IgG were hypersensitive to mechanical stimulation. Immunohistochemical analysis revealed that IgG from FMS patients specifically labeled satellite glial cells and myelinated fibre tracts, as well as a small number of macrophages and endothelial cells in mouse dorsal root ganglia but not skin, muscle, spinal cord and brain. Our results demonstrate that fibromyalgia pain is caused by IgG autoantibodies that sensitize peripheral nociceptive afferents neurons and suggest that therapies that reduce patient IgG titres may be effective treatments of fibromyalgia pain. 


\section{MAIN}

Fibromyalgia syndrome (FMS) is a chronic pain condition characterized by widespread pain, augmented pain sensitivity to mechanical pressure and cold temperatures ${ }^{3-5}$, as well as fatigue and emotional distress ${ }^{6-8}$. The prevalence of FMS is approximately $2 \%^{9}$, and at least $80 \%$ of FMS patients are women. The prevalence rises to $10-30 \%$ among patients diagnosed with autoimmune rheumatological conditions ${ }^{10,11}$, and FMS is thus one of the most common chronic pain conditions. The etiology and pathophysiology of FMS are unknown and the current treatment strategies for FMS rely mainly on life style changes, physical exercise and drug therapy with antidepressants and anticonvulsants ${ }^{1}$. Unfortunately, the modest efficacy of the available therapies in most patients leaves an enormous unmet clinical need. The animal models that have been used for experimental studies of FMS have uncertain translational relevance, and rely on local repeated intramuscular injections of acid $^{12}$ or systemic depletion of monoamines by reserpine treatment ${ }^{13}$. Consequently, the development of novel, mechanism-based therapies has been hampered by the limited understanding of the basis of FMS.

The increased polymodal pain sensitivity experienced by FMS patients ${ }^{14,15}$ is associated with altered pain processing in the central nervous system ${ }^{2}$, dysfunctional descending pain modulation ${ }^{16,17}$, and structural and functional changes in the brain ${ }^{18-20}$. FMS is also associated with abnormalities in peripheral sensory afferents, such as spontaneous ectopic activity and sensitization of C-fibers, and loss of epidermal innervation ${ }^{21,22}$. Furthermore, elevated levels of proinflammatory cytokines ${ }^{23,24}$, and reduced levels of anti-inflammatory cytokines ${ }^{25,26}$ have been detected in FMS patients, suggesting that inflammatory processes may be engaged. These observations, together with the markedly increased prevalence of FMS among patients with autoimmune rheumatological conditions ${ }^{10,11}$, led us to hypothesize that FMS may have an autoimmune basis. Here we have investigated the possibility that IgG autoantibodies cause FMS pain by examining whether sensory abnormalities can be transferred to mice by administration of IgG purified from FMS patients. 
We administered IgG purified from the serum of individual FMS patients and healthy control (HC) subjects to female mice by intraperitoneal injection for 4 consecutive days ( $8 \mathrm{mg}$ per day), a dose regimen based on the original studies identifying myasthenia gravis as an autoantibody mediated disorder ${ }^{27,28}$. Since FMS patients regularly report pressure hypersensitivity, we examined paw withdrawal thresholds in mice using the Randall-Selitto paw-pressure test following IgG transfer. IgG from each of the 8 patients (supplementary table 1), but not from any of the 6 healthy control subjects, rapidly produced mechanical hypersensitivity (Fig. 1a, e, g). In addition to pressure sensitivity, patients frequently report that pain is exacerbated by cold temperatures, and quantitative sensory testing has demonstrated an increased cold pain sensitivity in $\mathrm{FMS}^{4,15}$. In good agreement with patient observations, administration of IgG from FMS patients gave rise to a significantly increased sensitivity to noxious cold in mice (Fig.1b, $f, h$ ). Both mechanical and cold hypersensitivities were established within 24-48 hours after the first injection and maintained for at least about a week after the final IgG injection. FMS IgG also generated hypersensitivity to stimulation with calibrated von Frey filaments, a widely used test of mechanical nociception in mice (Fig. 1C). Fibromyalgia pain is widespread, and we therefore examined the pressure sensitivity of the thigh (Fig. 1d) using the Randall-Selitto device, to determine whether FMS IgG affects the pressure sensitivity of sites other than the hind paw in mice. In this test, FMS IgG produced significant mechanical hypersensitivity in the thigh compared to treatment with IgG from HC. The observed hypersensitivities produced by IgG preparations from different FMS patients showed similar amplitudes (Fig. 1e, f) and time courses, which is reflected in the plot showing the averaged mechanical and cold sensitivities produced by IgG from 8 individual FMS patients and $6 \mathrm{HC}$ subjects over 10 days (Fig. $1 \mathrm{~g}$, h). These results demonstrate that the transfer of sensitivities from FMS subjects to mice is reproducible across multiple FMS subject, strongly suggesting that an antibody-dependent mechanism may underlie FMS pain.

We next compared the effects of FM patient IgG with IgG-depleted patient serum to assess whether immunoglobulins and serum components other than IgG may contribute to pain and hypersensitivity in patients (Fig.1i, I). As expected, IgG from a single patient produced marked cold and mechanical 
hypersensitivities, whereas the IgG-depleted serum from the same patient was without any effect (Fig.1i, j).

To determine whether autoantibodies are responsible for pain and painful hypersensitivities in a wider and regionally distinct cohort of FMS patients, we examined the effects of transferring IgG preparations pooled from 20 FMS patients and 20 healthy control subjects (Supplementary table 1). As expected, FMS patients displayed markedly higher pain ratings on a visual-analogue scale (VAS; Fig. 2a) and a significantly increased pressure pain sensitivity compared to healthy control subjects (Fig. 2b). Administration of pooled IgG to mice (two separate experiments, each using IgG pooled from separate groups of 10 patients and 10 control subjects) significantly increased the sensitivity in the paw-pressure test compared to administration of pooled IgG from control subjects (Fig. 2c). In these experiments we used the same dose regimen as above (8mg of IgG on four consecutive days), but since the IgG from each individual donor was diluted 10-fold, the observed pro-nociceptive effect demonstrates that IgG from different patients have additive activity. Similar to our observations with IgG from individual patients, the pooled FMS IgG also elicited hypersensitivity to cold (Fig. 2d) and to stimulation with von Frey filaments (Fig. 2e). To determine the duration of the IgG-mediated hypersensitivity we studied mechanical thresholds in mice treated with IgG pooled from patients or control subjects for one month. The onset of mechanical hypersensitivity produced by pooled FMS IgG followed the same time course as seen with IgG from individual donors and resolved fully about 2.5 weeks after cessation of IgG administration (Fig. 2f). This observation indicates that the painful hypersensitivities produced by FMS IgG are reversible and that sensory abnormalities resolve when the levels of pathogenic IgG are reduced sufficiently. Our results thus strongly suggest that therapies which reduce patient IgG levels may be effective treatments for FMS pain.

\section{Sensitization of nociceptors}


Microneurography studies of FMS patients have demonstrated sensitization and ectopic impulse discharge in nociceptive $\mathrm{C}$-fibres ${ }^{21}$. To determine whether the sensory abnormalities demonstrated in mice in vivo following administration of FMS patient IgG could be explained by functional modification of peripheral nociceptors, we next examined the electrophysiological properties of single afferent nerve fibre units in skin-saphenous nerve preparations from IgG treated mice. Single units were classified according to their conduction velocity and mechanical response threshold, as described previously ${ }^{29,30}$.

We stimulated the receptive fields of $A \delta$ - and C-mechanosensitive nociceptors (AM and CM fibers) mechanically and compared the responses in preparations from mice after 4 days of treatment with IgG from either HC or FMS patients (Fig. 3). Both AM and CM fibers in preparations from mice treated with FMS IgG responded to mechanical stimulation with a significantly increased number and frequency of action potentials compared to preparations from HC IgG treated mice (Fig. 3a-d). The increased impulse activity evoked by mechanical stimulation is consistent with the C-fibre sensitization observed in FMS patients using microneurography ${ }^{21}$ and demonstrates that patient autoantibodies produce a heightened nociceptor activity that is maintained in the absence of the central nervous system. Since we had observed a robust behavioral cold hypersensitivity in mice treated with FMS IgG, we next examined whether this phenotype was accompanied by functional

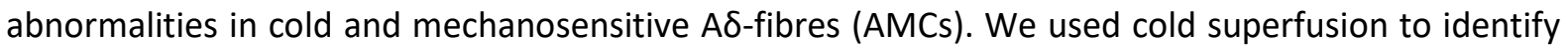
the receptive fields of AMCs and thereafter challenged these with a $60 \mathrm{~s}$ cooling ramp (from $32^{\circ} \mathrm{C}$ to 5 $9^{\circ} \mathrm{C}$ ). Cooling evoked a significantly increased number of action potentials in AMCs in preparations from mice treated with FMS IgG compared to HC IgG (Fig.3 e, f; FMS median 82, interquartile range 41-353; HC 22, 3-52), whereas as the cold activation thresholds did not differ significantly (Fig.3 g). Analysis of the temporal impulse pattern demonstrated that AMCs from FMS IgG treated mice discharged action potentials at a higher rate throughout the cooling ramp, consistent with a heightened excitability (Fig. $3 \mathrm{~h}$ ). 
Immunohistochemical localization of human IgG

Immunohistochemical analysis of tissues from mice that had been injected with FMS IgG using antihuman IgG antibodies revealed robust staining in dorsal root ganglia (DRG; Fig. 3a), while no specific immunoreactivity was observed in the spinal cord (Fig.3 c, Supp. Fig. 1a). In contrast, IgG from control subjects generated only low levels of immunoreactivity in DRGs (Fig. 3a, b). FMS IgG was also reliably detected in DRG, but neither in brain nor spinal cord tissue by western blot analysis (Fig. 3c). The FMS patient IgG was primarily located to satellite glial cells (visualized by glutamine synthase) and to fibre tracts entering the DRG, as well as a number of Iba1 positive macrophages and CD31 positive blood vessels (Fig 3d). FMS patient IgG did not colocalize with DRG neurons (visualized by NeuN). In addition to the specific localization of FMS IgG to satellite glial cells, we noted signs of enhanced satellite cell activity, based on changes in GFAP immunoreactivity, in mice injected with FMS IgG compared to HC IgG injected controls (Fig 3e-g). In contrast, no changes in astrocyte (GFAP; Supp. Fig. 1b-d) or microglia (Iba1; Supp. Fig. 1e-g) reactivity were observed in the spinal cord. In combination with the lack of FMS IgG in the brain of spinal cord, these observations strongly suggest that the pronociceptive actions of FMS IgG were peripheral. Future investigations will determine whether an action of IgG on satellite cells is responsible for FMS pain and elucidate the molecular mechanisms underlying the neuronal hypersensitivities. It is clear, however, that satellite glial cells and sensory neurons can be electrically coupled and that satellite cells can influence neuronal excitability ${ }^{31-33}$.

Here we identify FMS as an autoimmune pathology in which IgG purified from FMS patients evokes painful hypersensitivity by sensitizing peripheral nociceptors when transferred to mice. A peripheral origin of pain and tenderness agrees well with earlier reports of peripheral sensory abnormalities in patients $^{5,15,21,22}$. A chronically heightened noxious peripheral input may be expected to generate altered patterns of activity in the $\mathrm{CNS}^{20}$. Identification of FMS as an autoimmune disease, rather than 
an unexplained functional somatic syndrome, will transform future fibromyalgia research and facilitate development of mechanism-based therapeutic interventions. Our results suggest that therapies that reduce the IgG titre in patients, or which prevent the functional consequences of the pathological IgG, will be effective therapies for fibromyalgia.

Acknowledgements: This work was supported by the Medical Research Council MR/S003428/1 (DAA, SB and AG), Versus Arthritis 21544 (DAA and AG) and 21543 (SB) the Pain Relief Foundation (DAA and AG), the Swedish Research Council (CIS), Knut and Alice Wallenberg Foundation (CIS), the Karolinska Institute Foundations (CMU), the EU Projects FP7-Health-2013-Innovation-1602919-2 (CIS), European Union Seventh Framework Programme (FP7/2007-2013) under grant agreement no 602919, the IASP John J. Bonica fellowship (EK) and by a generous donation from Leif Lundblad and family (CIS, EK). 


\section{FIGURE LEGENDS}
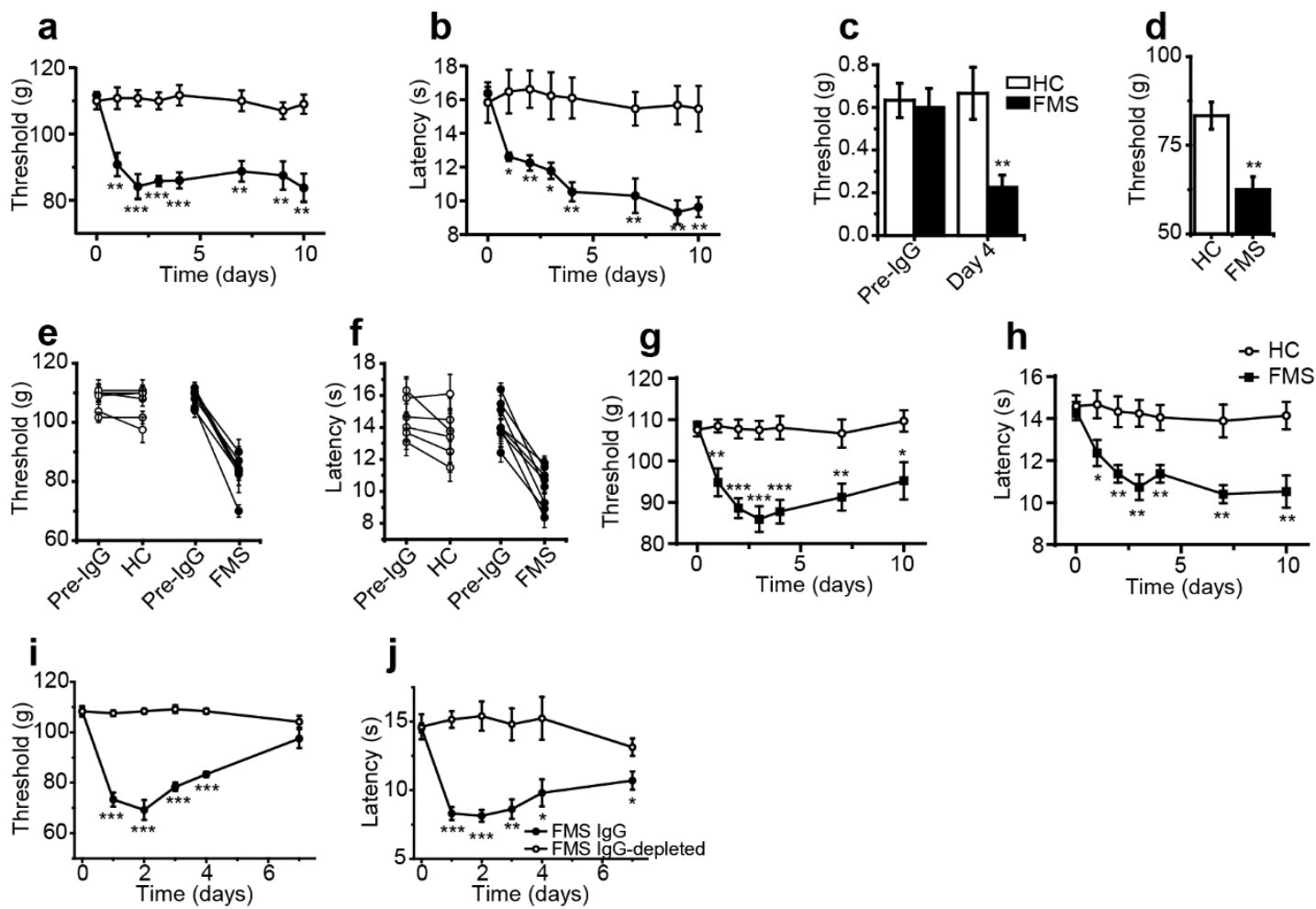

Figure 1. Passive transfer of hypersensitivities from fibromyalgia patients to mice.

Administration of IgG from an FMS patient reduced the paw withdrawal threshold in the paw-pressure test (a), the withdrawal latency in the cold-plate test (b), the response threshold to stimulation with von Frey filaments $(\mathbf{c})$, and the withdrawal threshold to mechanical pressure of the thigh (d), compared to administration of IgG from healthy control subjects. Administration of purified IgG from each of $8 \mathrm{FMS}$ patients evoked mechanical (e; $\mathrm{p}$ between 0.02 and $2 \times 10^{-5}$ ) and cold hypersensitivity (f, $p$ between 0.05 and $2 \times 10^{-4}$ ), whereas 6 preparations from HC did not (all $p>0.25$ in the paw pressure test and $p>0.15$ in the cold plate test). Paw withdrawal threshold or latency before injection of IgG and at the time of maximum sensitivity during the 7 days following $\lg G$ injections are shown in $\mathbf{e}, \mathbf{f}$. Mean sensitivity in the paw-pressure test (g) and cold plate test (h) following administration of IgG from 8 patients and 6 control subjects (each experiment performed as in a, b). IgG from a single patient, but not the IgG-depleted serum from the same patient, produced mechanical (i) and cold (j) hypersensitivity. Data points are mean \pm SEM of $n=6$ mice in a-d. Data points in e-f are mean \pm SEM 
of 8 (FMS) or 6 (HC) experiments, each performed with $n=4-6$ mice per treatment. ${ }^{*} \mathrm{P}<0.05,{ }^{* *} \mathrm{P}<0.01$, $* * * \mathrm{P}<0.001, \mathrm{FMS}$ IgG compared to HC IgG, unpaired two-tailed t-test.

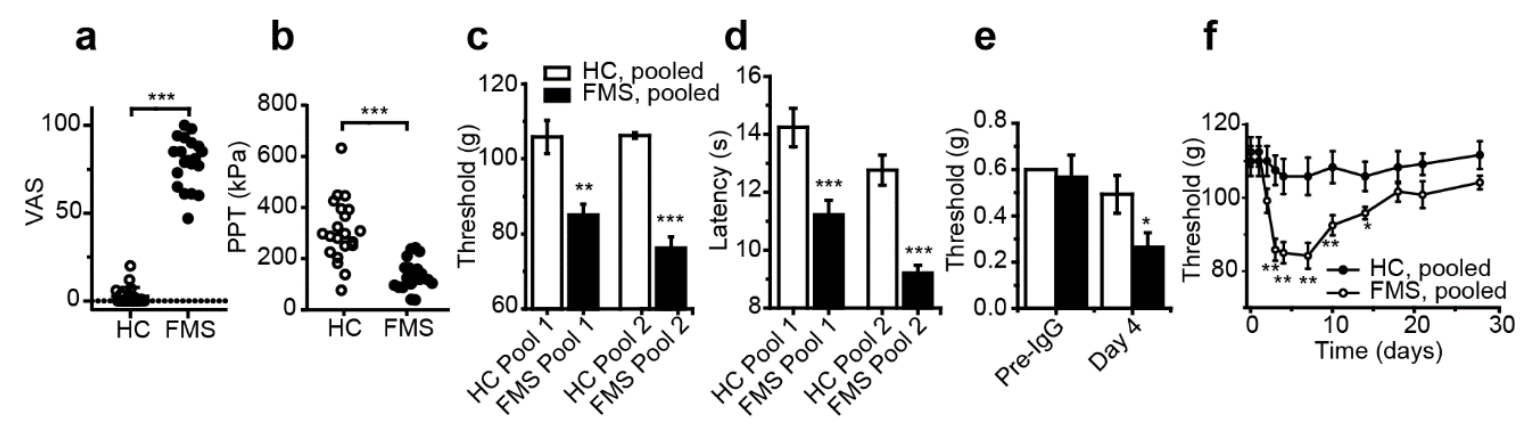

Figure 2. Passive transfer of hypersensitivity by pooled IgG. Visual analogue pain scores (a) and pressure pain thresholds (b) reported by 20 FMS patients and 20 healthy control subjects. Administration of IgG from 2 pools of 10 FMS patients produced mechanical (c), cold (d) and tactile (von Frey, pool 1, e) hypersensitivity in mice compared to 2 pools of HC IgG (each pool from 10 subjects). Time course of mechanical hypersensitivity following administration of IgG pooled from 10 FMS patients and $\mathrm{HC}(\mathrm{f}) .{ }^{*} \mathrm{P}<0.05,{ }^{*} \mathrm{P}<0.01,{ }^{* *} \mathrm{P}<0.001, \mathrm{FMS} \operatorname{IgG}$ compared to HC IgG, unpaired twotailed t-test or Mann-Whitney U-test (a, b) or unpaired two-tailed t-test (c-f). 
a
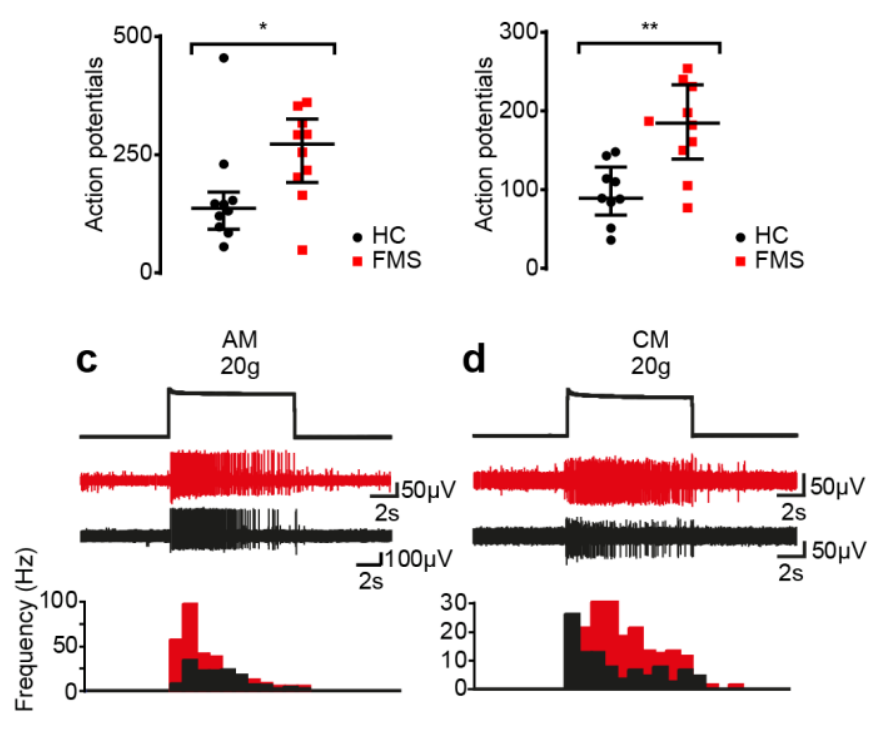

b

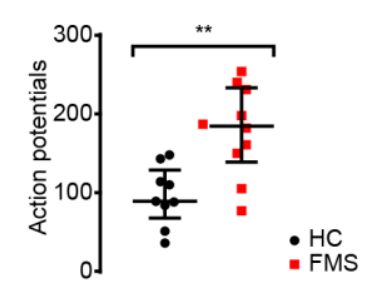

e

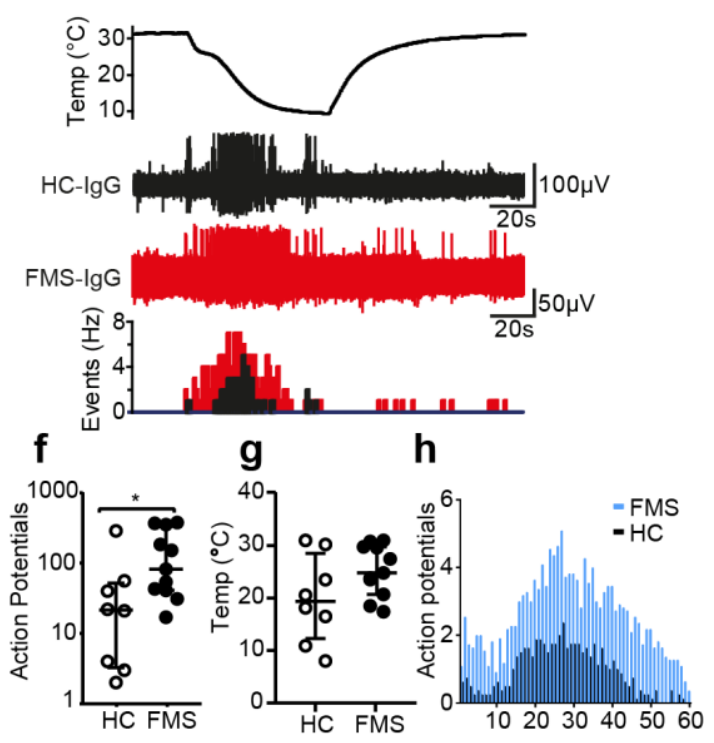

Figure 3. FMS IgG sensitizes nociceptors. The number of action potentials elicited by a force step (20g, 10s) was significantly increased in A $\delta$ - (a) and C-mechanonociceptors (b) in preparations from mice treated with FMS IgG compared to HC IgG. Typical recordings of A $\delta$ - ("AM") and Cmechanonociceptors ("CM") in FMS and HC preparations (c-d). The lower panels show the increased action potential frequency in FMS IgG treated preparations compared to HC IgG. Typical recordings of cold-sensitive AM fibres (AMCs) in preparations from mice treated with FMS or HC IgG (e). The number of cooling-evoked action potentials ( $\mathbf{f}$ ) the temperature activation thresholds ( $g$ ) in AMCs from mice treated with FMS IgG compared to HC IgG. The average impulse pattern during 60 s cooling ramps demonstrate an increased discharge rate in AMCs from FMS IgG treated mice. Line and whiskers indicate median and interquartile range. ${ }^{*} \mathrm{P}<0.05,{ }^{*} \mathrm{P}<0.01$, FMS IgG compared to HC IgG, MannWhitney U-test. 

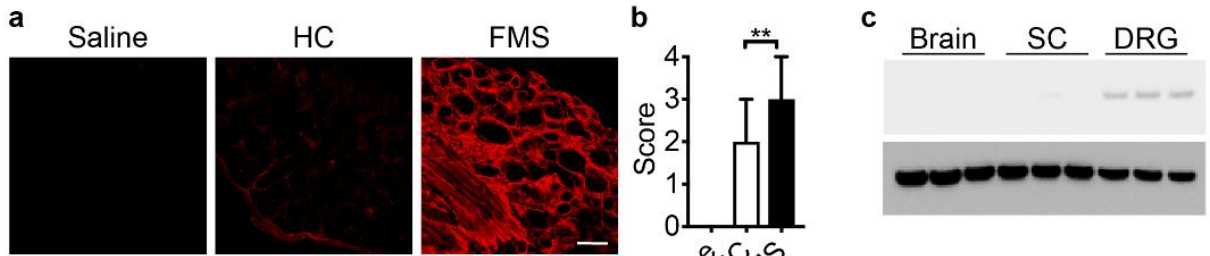

$5^{2 n^{2}}+N^{5}$
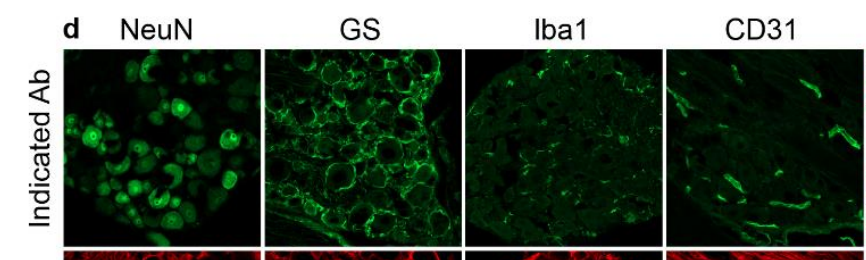

MBP
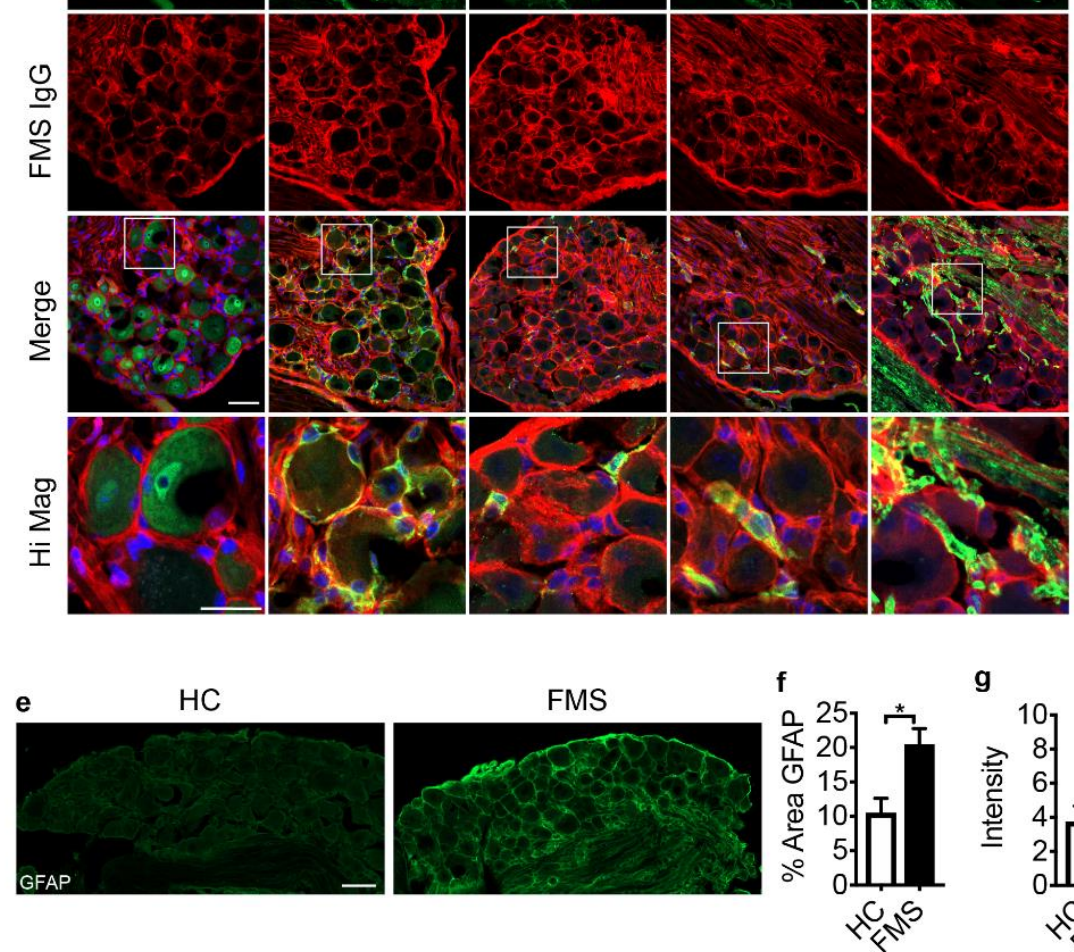

g

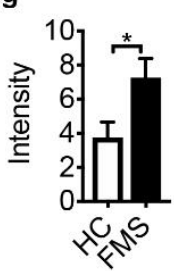

Figure 4. FMS IgG accumulates in the DRG and binds satellite glial cells. FMS IgG, but not HC IgG accumulates in the dorsal root ganglia (DRG) 14 days after the first IgG injection (a, b). Western blot analysis detected FMS IgG in the DRG but little to no IgG in spinal cords (SC) or brains (c) at the last day of IgG injection (day 4). FMS IgG immunoreactivity does not colocalize with neurons (NeuN positive cells) but does colocalize with satellite cells (glutamate synthase (GS) expressing cells), some macrophages (Iba1 expressing cells) and blood vessels (CD31 expressing cells) and myelinated fibre tracts (myelin basic protein (MBP) staining) within but not outside the DRG compartment (d). DRGs 
from FMS IgG injected mice have increased GFAP immunoreactivity, which is indicative of increased satellite glial cell activity, compared to HC injected mice (e) when the \% area of GFAP immunoreactivity (f) and GFAP mean pixel intensity (g) are quantified. Human IgG immunoreactivity was quantified using a custom 0 (no $\lg G$ ) to 4 (very strong $\lg G$ signal), $n=9-10$, data points are presented as median $\pm 95 \%$ C.I. GFAP data points are mean \pm SEM, $n=5-6 . *$ indicates $p<0.05, * *$ indicates $p<0.01$ compared to $H C$ IgG, Kruskal-Wallis test followed by a Mann-Whitney test (b) or t-test (f, g). Scale bars indicate $50 \mu \mathrm{m}$, except the high magnification image scale bar which indicates $25 \mu \mathrm{m}$. 

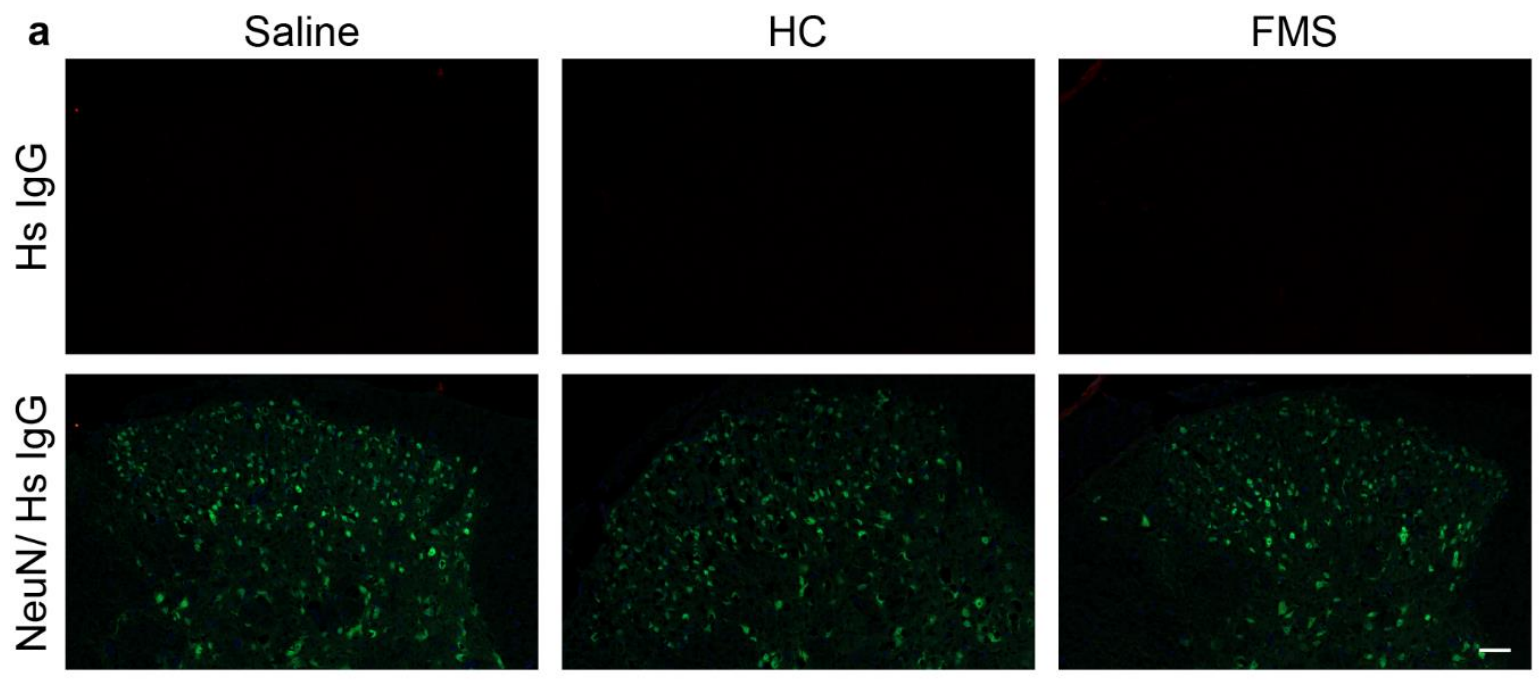

b

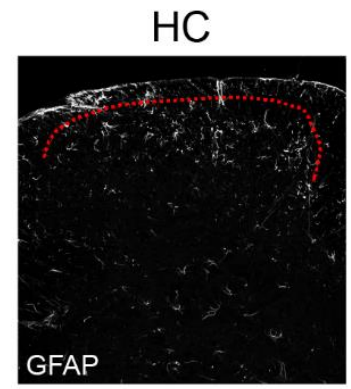

e

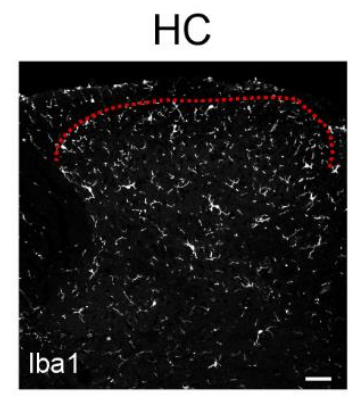

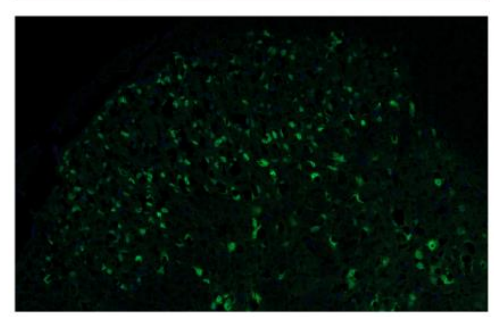

FMS

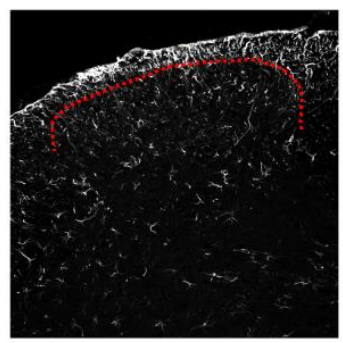

FMS

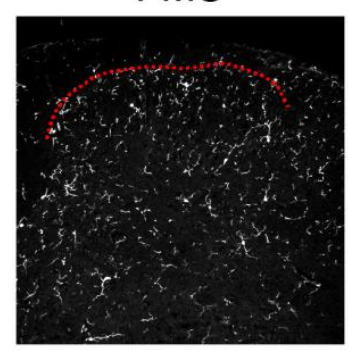

C

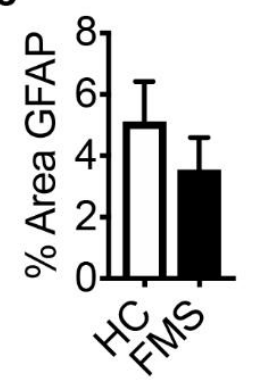

f

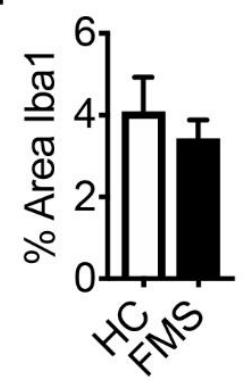

d

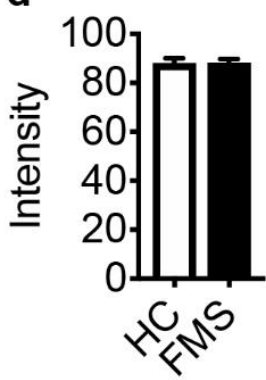

g

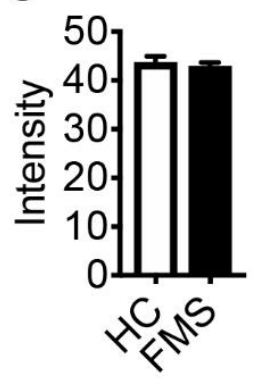

Supplemental Figure 1. FMS IgG does not accumulate in the spinal cord or activate dorsal horn glia.

Neither HC IgG nor FMS IgG accumulates in the dorsal horn of the spinal cord (a). Sections were probed with an anti-Hs (human) IgG antibody (top row) and anti-NeuN antibody (bottom row, merged with anti-Hs IgG, a). FMS IgG did not change the percent area of GFAP immunoreactivity of GFAP intensity in the dorsal horn compared to HC IgG (b-d), suggesting that FMS IgG does not alter astrocyte activity. Iba1 percent area of immunoreactivity and intensity were also unchanged by FMS IgG compared to HC IgG (f-g), suggesting that FMS IgG does not alter microglia activity. Red dotted lines indicate dorsal edge of lamina I of the dorsal horn. Data points are mean \pm SEM, $n=5-6$. Scale bars are $50 \mu \mathrm{m}$. 


\section{REFERENCES}

1. Macfarlane, G. J. et al. EULAR revised recommendations for the management of fibromyalgia. Annals of the Rheumatic Diseases 76, 318-328 (2017).

2. Sluka, K. A. \& Clauw, D. J. Neurobiology of fibromyalgia and chronic widespread pain. Neuroscience 338, 114-129 (2016).

3. Tampin, B., Briffa, N. K. \& Slater, H. Self-reported sensory descriptors are associated with quantitative sensory testing parameters in patients with cervical radiculopathy, but not in patients with fibromyalgia. Eur J Pain 17, 621-633 (2013).

4. Hurtig, I. M., Raak, R. I., Kendall, S. A., Gerdle, B. \& Wahren, L. K. Quantitative sensory testing in fibromyalgia patients and in healthy subjects: identification of subgroups. Clin J Pain 17, 316-322 (2001).

5. Blumenstiel, K. et al. Quantitative sensory testing profiles in chronic back pain are distinct from those in fibromyalgia. Clin J Pain 27, 682-690 (2011).

6. Arnold, L. M. et al. Comorbidity of fibromyalgia and psychiatric disorders. J Clin Psychiatry 67, 1219-1225 (2006).

7. Thieme, K., Turk, D. C. \& Flor, H. Comorbid depression and anxiety in fibromyalgia syndrome: relationship to somatic and psychosocial variables. Psychosom Med 66, 837-844 (2004).

8. Nicholas, M. et al. The IASP classification of chronic pain for ICD-11: chronic primary pain. Pain 160, 28-37 (2019).

9. Queiroz, L. P. Worldwide epidemiology of fibromyalgia. Curr Pain Headache Rep 17, 356 (2013).

10. Perrot, S. et al. Patient phenotypes in fibromyalgia comorbid with systemic sclerosis or rheumatoid arthritis: influence of diagnostic and screening tests. Screening with the FiRST questionnaire, diagnosis with the ACR 1990 and revised ACR 2010 criteria. Clin. Exp. Rheumatol. 35 Suppl 105, 35-42 (2017).

11. Fitzcharles, M.-A., Perrot, S. \& Häuser, W. Comorbid fibromyalgia: A qualitative review of prevalence and importance. Eur J Pain 22, 1565-1576 (2018).

12. Yen, L.-T., Hsieh, C.-L., Hsu, H.-C. \& Lin, Y.-W. Targeting ASIC3 for Relieving Mice Fibromyalgia Pain: Roles of Electroacupuncture, Opioid, and Adenosine. Sci Rep 7, 46663 (2017).

13. Nagakura, Y., Oe, T., Aoki, T. \& Matsuoka, N. Biogenic amine depletion causes chronic muscular pain and tactile allodynia accompanied by depression: A putative animal model of fibromyalgia. Pain 146, 26-33 (2009).

14. Kosek, E., Ekholm, J. \& Hansson, P. Sensory dysfunction in fibromyalgia patients with implications for pathogenic mechanisms. Pain 68, 375-383 (1996).

15. Berglund, B., Harju, E. L., Kosek, E. \& Lindblom, U. Quantitative and qualitative perceptual analysis of cold dysesthesia and hyperalgesia in fibromyalgia. Pain 96, 177-187 (2002). 
16. Kosek, E. \& Hansson, P. Modulatory influence on somatosensory perception from vibration and heterotopic noxious conditioning stimulation (HNCS) in fibromyalgia patients and healthy subjects. Pain 70, 41-51 (1997).

17. Jensen, K. B. et al. Evidence of dysfunctional pain inhibition in Fibromyalgia reflected in rACC during provoked pain. Pain 144, 95-100 (2009).

18. Jensen, K. B. et al. Overlapping structural and functional brain changes in patients with longterm exposure to fibromyalgia pain. Arthritis Rheum. 65, 3293-3303 (2013).

19. Schrepf, A. et al. Endogenous opioidergic dysregulation of pain in fibromyalgia: a PET and fMRI study. Pain 157, 2217-2225 (2016).

20. Kaplan, C. M. et al. Functional and neurochemical disruptions of brain hub topology in chronic pain. Pain (2019). doi:10.1097/j.pain.0000000000001480

21. Serra, J. et al. Hyperexcitable C nociceptors in fibromyalgia. Ann. Neurol. 75, 196-208 (2014).

22. Üçeyler, N. et al. Small fibre pathology in patients with fibromyalgia syndrome. Brain 136, 1857-1867 (2013).

23. Kadetoff, D. \& Kosek, E. Evidence of reduced sympatho-adrenal and hypothalamic-pituitary activity during static muscular work in patients with fibromyalgia. J Rehabil Med 42, 765-772 (2010).

24. Bäckryd, E., Tanum, L., Lind, A.-L., Larsson, A. \& Gordh, T. Evidence of both systemic inflammation and neuroinflammation in fibromyalgia patients, as assessed by a multiplex protein panel applied to the cerebrospinal fluid and to plasma. J Pain Res 10, 515-525 (2017).

25. Uçeyler, N. et al. Reduced levels of antiinflammatory cytokines in patients with chronic widespread pain. Arthritis Rheum. 54, 2656-2664 (2006).

26. Uçeyler, N., Häuser, W. \& Sommer, C. Systematic review with meta-analysis: cytokines in fibromyalgia syndrome. BMC Musculoskelet Disord 12, 245 (2011).

27. Toyka, K. V., Brachman, D. B., Pestronk, A. \& Kao, I. Myasthenia gravis: passive transfer from man to mouse. Science 190, 397-399 (1975).

28. Toyka, K. V. et al. Myasthenia gravis. Study of humoral immune mechanisms by passive transfer to mice. N. Engl. J. Med. 296, 125-131 (1977).

29. Zimmermann, K. et al. Phenotyping sensory nerve endings in vitro in the mouse. Nat Protoc 4, 174-196 (2009).

30. Vastani, N. et al. Impaired Nociception in the Diabetic Ins2+/Akita Mouse. Diabetes 67, 1650-1662 (2018).

31. Spray, D. C. et al. Gap junction mediated signaling between satellite glia and neurons in trigeminal ganglia. Glia (2019). doi:10.1002/glia.23554

32. Ji, R.-R., Berta, T. \& Nedergaard, M. Glia and pain: is chronic pain a gliopathy? Pain $\mathbf{1 5 4}$ Suppl 1, S10-28 (2013).

33. Xu, M. et al. Pain and the immune system: emerging concepts of IgG-mediated autoimmune pain and immunotherapies. J. Neurol. Neurosurg. Psychiatry (2018). doi:10.1136/jnnp-2018-318556 


\section{METHODS}

\section{Patient samples}

Serum samples for individual testing were derived from UK patients managed for their Fibromyalgia at a department of pain medicine, or from healthy controls (Ethics North-West Haydock, Ref: 15/NW/0467). Pooled samples were obtained from Swedish patients or healthy controls responding to a study advert (approved by the local ethical committee 2011/2036-31/1). Individual informed consent was obtained from all patients. All patients had been examined by a Consultant rheumatologist, and UK patients had additionally been examined by a Consultant in pain medicine. We purified serum-IgG from 28 FMS patients and 26 healthy control subjects (HC). All patients fulfilled both 1990 and 2011 ACR diagnostic criteria for fibromyalgia ${ }^{1,2}$. Most patients (26/28) and all of the pooled sample donors were unaffected by other sensory or rheumatological conditions. Most patient donors (27/28) were women. The donors' demographics and disease characteristics are provided in Supplemental tables 1-2.

\begin{tabular}{|l|l|l|l|l|}
\hline & Age; \%female & Duration (years) & Pain intensity & Tender point count \\
\hline UK sample, $n=8$ & $46(19-58) ; 88$ & $2.5(1.3-18)$ & $8.1 \pm 0.8(6.5-9)$ & $15(12-18)$ \\
\hline Swedish sample, & $49(35-58) ; 100$ & $11(3-15)$ & $79 \pm 14(47-100)$ & $17(11-18)$ \\
\hline
\end{tabular}

Supplemental table 1. Patient characteristics. Age - mean (range), duration of FMS pain - median (interquartile range); pain intensity - average pain intensity over the past week \pm SD (range); UK sample-numeric rating scale pain score on an 11-point scale (0-10), Swedish sample - visual analogue scale pain score (0-100), with 10, 100 = 'worst pain imaginable'; tender point count - average (range) number of defined points which are tender to pressure out of a maximal $18 .^{1}$ 


\section{Purification of immunoglobulins}

IgG for individual testing was purified as described previously ${ }^{3}$, using protein $\mathrm{G}$ beads (Sigma-Aldrich, Gillingham, UK). Serum was diluted 1:3 with Hartmann's solution, passed through a protein G column, and the bound IgG was eluted using $100 \mathrm{mM}$ glycine $\mathrm{pH} 2.3$; the $\mathrm{pH}$ was adjusted to 7.4 using $1 \mathrm{M}$ Tris $\mathrm{pH} 8$ and then dialyzed overnight at $4^{\circ} \mathrm{C}$ in Hartmann's using a $10 \mathrm{kDa}$ dialysis membrane (Fisher Scientific, Loughborough, UK). The concentration of IgG present after dialysis was determined using a modified Lowry assay (DC protein assay, BioRad, Hemel Hempstead, UK) and adjusted by dilution with Hartmann's or by dialysis against a sucrose solution (Sigma-Aldrich). Finally, the IgG solution was sterile filtered using syringe-driven $0.2 \mu \mathrm{m}$ filter units (Millipore, Watford, UK), stored at $4^{\circ} \mathrm{C}$ and used within 3 months. Sera for pooled sample testing was purified using HiTrap Protein G HP columns (GE

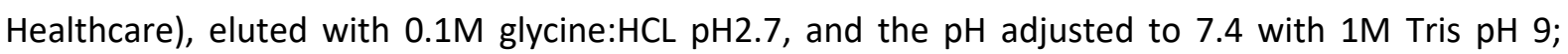
consequently samples were dialysed against PBS, concentration adjusted, stored at $-20 \mathrm{C}$ and later thawed, pooled, and concentration-adapted using PBS pre-wet concentration columns (Pall Corporation Macrosep 10K); concentration measurements were always with Nanodrop 2000 (Fisher Scientific).

\section{Animals}

Behavioral experiments were carried out according to the U.K. Home Office Animal Procedures (1986) Act. All procedures were approved by the King's College London Animal Welfare and Ethical Review Body and conducted under a UK Home Office Project License. Behavioural experiments were performed on female C57BI/6J mice (8-10 weeks old) obtained from Envigo UK Ltd., Bicester, UK, housed in a temperature-controlled environment with a $12 \mathrm{~h}$ light/dark cycle with access to food and water ad libitum. Immunofluorescence experiments were performed on 4-month old female BALB/C mice (Janvier, France) and were approved by the local ethical committee (4945-2018). Mice were injected intraperitoneally with $8 \mathrm{mg}$ of IgG from healthy control subjects or FMS patients on 4 consecutive days. 


\section{Behavioral studies}

Before any nociceptive testing, mice were kept in their holding cages to acclimatize (10-15 $\mathrm{min})$ to the experimental room. Mice were randomized between cages and the experimenter blinded to their treatment.

The Randall-Selitto paw-pressure test was performed using an Analgesymeter (Ugo-Basile, Italy). The experimenter lightly restrained the mouse and applied a constantly increasing pressure stimulus to the dorsal surface of the hind paw using a blunt conical probe. The nociceptive threshold was defined as the force in grams at which the mouse withdrew its paw ${ }^{4}$. A force cut-off value of $150 \mathrm{~g}$ was used to avoid tissue injury.

Tactile sensitivity was assessed using von Frey filaments (0.008-2 g) according to Chaplan's up-down method $^{5}$. Animals were placed in a Perspex chamber with a metal grid floor allowing access to their plantar surface and allowed to acclimatize prior to the start of the experiment. The von Frey filaments were applied to the plantar surface of the hind paw with enough force to allow the filament to bend, and held static for approximately $2-3 \mathrm{~s}$. The stimulus was repeated up to 5 times at intervals of several seconds, allowing for resolution of any behavioral responses to previous stimuli. A positive response was noted if the paw was sharply withdrawn in response to filament application or if the mouse flinched upon removal of the filament. Any movement of the mouse, such as walking or grooming, was deemed an unclear response, and in such cases the stimulus was repeated. If no response was noted a higher force hair was tested and the filament producing a positive response recorded as the threshold.

Thermal sensitivity was assessed using a cold-plate (Ugo Basile, Milan). Paw withdrawal latencies were determined with the plate set at $10{ }^{\circ} \mathrm{C}$. The animals were lightly restrained (scruffed) and the left hind paw was placed onto the surface of the plate $e^{6,7}$. The latency to withdrawal of the paw was recorded as the endpoint. A maximum cut-off of 30 seconds was used for each paw. 


\section{Skin-nerve recording}

Mice were killed by cervical dislocation and the hind paw was shaved prior to dissection of the isolated skin-nerve preparation. The saphenous nerve and the shaved skin of the hind limb were placed in a recording chamber at $32{ }^{\circ} \mathrm{C}$. The chamber was perfused with a gassed $\left(95 \% \mathrm{O}_{2}\right.$ and $\left.5 \% \mathrm{CO}_{2}\right)$ prewarmed synthetic interstitial fluid (SIF): $108 \mathrm{mM} \mathrm{NaCl}, 3.5 \mathrm{mM} \mathrm{KCl}, 0.7 \mathrm{mM} \mathrm{MgSO}_{4}, 26.2 \mathrm{mM} \mathrm{NaCO}_{3}, 1.65 \mathrm{mM}$ $\mathrm{NaH}_{2} \mathrm{PO}_{4}, 1.53 \mathrm{mM} \mathrm{CaCl}, 9.6 \mathrm{mM}$ sodium gluconate, $5.55 \mathrm{mM}$ glucose and $7.6 \mathrm{mM}$ sucrose. The skin was placed inside up (corium side up) and pinned down using insect pins ( $0.2 \mathrm{~mm}$ diameter) in the organ bath to allow access to the receptive fields. The saphenous nerve was placed through a small gap from the organ bath to an adjacent recording chamber on a mirror platform. The desheathed saphenous nerve was covered with paraffin oil for electrical isolation and dissected fine nerve filaments were placed on a fine gold wire-recording electrode using a microscope $e^{8,9}$.

\section{Conduction velocity}

The saphenous nerve was divided into progressively thinner filaments until a single unit could be isolated in response to stimulation of the receptive field with a glass rod. The electrical latency of identified units (Digitimer DS2, Digitimer Ltd, Welwyn Garden City, UK) was used to determine the conduction velocity and to categorize units as $A \beta$ (velocity $>10 \mathrm{~m} / \mathrm{s}), A \delta(1.2<$ velocity $<10 \mathrm{~m} / \mathrm{s})$ or Cfibers $(0<$ velocity $<1.2 \mathrm{~m} / \mathrm{s})$.

\section{Mechanical stimulation}

A computer-controlled stimulating probe, equipped with a force transducer, was used to deliver mechanical stimuli to the most sensitive point of a receptive field (Avere Solutions UG, Erlangen, Germany). The number of action potentials elicited by stimulation with a 10 s force step of $20 \mathrm{~g}$ was determined in $\mathrm{A}$ - and $\mathrm{C}$-mechanonociceptors ( $\mathrm{AM}$ and $\mathrm{CM}$ fibers) in preparations from mice treated with FMS or HC IgG. Recording and analysis were done using Spike 2 (Cambridge Electronic Design, Cambridge, UK).

Immunofluorescence 
Mice were deeply anesthetized with isoflurane and perfused with PBS, followed by $4 \%$ PFA. The lumbar spinal cord and lumbar dorsal root ganglia were collected, post fixed for 24 hours in 4\% PFA, cryoprotected in $20 \%$ sucrose and then embedded in optimal cutting temperature (OCT) compound (Sakura Finetek). Tissue was sectioned with a CryoStar NX70 cryostat (Thermo Scientific) at a thickness of $10 \mu \mathrm{m}$ and thaw mounted onto SuperFrost Plus slides (Thermo Scientific) and slides were stored at $-20^{\circ} \mathrm{C}$ until use. Prior to use slides were thawed for at least 30 minutes at room temperature and washed in PBS to remove excess OCT. Slides were blocked with PBS supplemented with $0.3 \%$ TritonX-100 and 3\% normal goat or donkey serum, depending on the secondary antibody and then incubated with primary antibodies diluted in PBS supplemented with $0.1 \%$ Triton X-100 and $1 \%$ normal goat or donkey serum. Slides were washed and incubated with appropriate secondary antibodies (indicated in supplementary table 2). For colocalization studies of human IgG with various cell-type markers the primary antibodies against the cell type markers were first added and then following washing the anti-human IgG antibody was co-incubated with the secondary antibodies against cell-type markers. Following washing, the slides were incubated with DAPI (1:20000), washed again and then cover slipped with Prolong Gold mounting media (Thermo Scientific).

Supplemental table 2. Antibodies used.

\begin{tabular}{|c|c|c|c|c|c|c|}
\hline \multicolumn{7}{|c|}{ Primary Antibodies } \\
\hline Target & Host & \multicolumn{2}{|c|}{ Manufacturer } & \multicolumn{2}{|c|}{ Catalogue \# } & Dilution \\
\hline GS & Rabbit & \multicolumn{2}{|c|}{ Abcam } & \multicolumn{2}{|c|}{ ab73593 } & $1: 500$ \\
\hline GFAP & Mouse & \multicolumn{2}{|c|}{ Millipore } & \multicolumn{2}{|c|}{ Mab360 } & $\begin{array}{l}1: 500 \text { (DRG) } \\
1: 1000(\mathrm{SC})\end{array}$ \\
\hline Iba1 & Rabbit & \multicolumn{2}{|l|}{ Wako } & \multicolumn{2}{|c|}{ 019-19741 } & $\begin{array}{l}1: 500 \text { (DRG) } \\
1: 1000 \text { (SC) }\end{array}$ \\
\hline $\begin{array}{l}\text { NeuN conj. } \\
\text { AF488 }\end{array}$ & Mouse & \multicolumn{2}{|c|}{ Millipore } & \multicolumn{2}{|c|}{ MAB377X } & 1:100 \\
\hline MBP & Rat & \multicolumn{2}{|c|}{ Abcam } & \multicolumn{2}{|c|}{550274} & $1: 500$ \\
\hline CD31 & Rat & BD Pha & gen & Ab73 & & $1: 500$ \\
\hline \multicolumn{7}{|c|}{ Secondary Antibodies } \\
\hline Target & Fluorophore & Host & \multicolumn{2}{|c|}{ Manufacturer } & Catalogue \# & Dilution \\
\hline Rabbit IgG & AF488 & Goat & \multicolumn{2}{|c|}{ ThermoFisher } & A11008 & $1: 250$ \\
\hline Human IgG & AF594 & Goat & \multicolumn{2}{|c|}{ ThermoFisher } & A11014 & $1: 200$ \\
\hline Mouse IgG & AF488 & Goat & \multicolumn{2}{|c|}{ ThermoFisher } & A11029 & $1: 250$ \\
\hline Rat IgG & AF488 & Goat & \multicolumn{2}{|c|}{ ThermoFisher } & A11006 & $1: 250$ \\
\hline
\end{tabular}


GS (glutamate synthase), GFAP (Glial fibrillary acidic protein), NeuN (Neuronal N), MBP (Myelin Basic Protein), AF (Alexa Fluor)

\section{Fluorescent microscopy and analysis}

DRGs were imaged using a confocal microscope (Zeiss LSM800) operated by LSM ZEN2012 (Zeiss) software. For each animal 5 DRG sections separated at least $50 \mu \mathrm{m}$ were analyzed and averaged. Human IgG accumulation was assessed by two blinded scorers using a 0-4 visual scoring system and the GFAP signal was analyzed in ImageJ as \% GFAP positive area and average GFAP pixel intensity. Spinal cords were imaged using a Nikon Eclipse TE300 fluorescent microscope with a Nikon Digital Sight DS-Fi1 camera. Iba1 and GFAP signal intensity were analysed by two blinded reviewers using a custom developed Python script. For each animal 5 spinal cord sections separated at least $50 \mu \mathrm{m}$ were analyzed and averaged and the data presented as mean \pm SEM. Representative spinal cord images were taken using a Zeiss LSM 800 confocal microscope.

\section{Western blot analysis}

Mice were deeply anesthetized with isoflurane and euthanized by decapitation. Brain, spinal cord and lumbar DRG were collected and flash frozen for later use. Protein was extracted from the tissue by sonication in an extraction buffer $(0.5 \%$ Triton X-100, $50 \mathrm{mM}$ Tris, $150 \mathrm{mM} \mathrm{NaCl}, 1 \mathrm{mM}$ EDTA and $1 \%$ SDS in water, pH 7.4). Protein concentration was analyzed using with the BCA Assay (Pierce). Samples were diluted in an LDS loading buffer (NuPage) and denatured with DTT at $95^{\circ} \mathrm{C}$. An equal amount of protein per sample was loaded into 2-12\% gradient NuPage Bis-Tris gels (Thermo Scientific) and protein was separated by electrophoresis. Protein was transferred to a nitrocellulose membrane using a dry transfer system (Thermo Scientific). The membrane was blocked with $5 \%$ non-fat milk powder (Biorad) in TBS.T for 1 hour at room temperature. The membrane was then probed with an anti-human HRP conjugated antibody (Santa Cruz) diluted in TBS.T with 5\% non-fat 
milk powder. SuperSignal West Pico Chemiluminescent Substrate (Thermo Scientific) was incubated with the membrane and protein was visualized using a Biorad ChemiDoc ${ }^{\mathrm{TM}}$ Image System.

\section{Reagents}

All buffers and salts were purchased from Sigma (Poole, UK), VWR (Lutterworth, UK) or Thermofisher (Waltham, MA, USA).

\section{Statistics}

Data are presented as individual data points or as mean \pm SEM and the number of animals or single units studied are indicated by $\mathrm{n}$. No data points have been removed from any of the datasets presented. Data from behavioral in vivo experiments were tested statistically by unpaired, two-tailed t-test, whereas patient data and electrophysiological data were tested by Mann-Whitney U-test. 


\section{REFERENCES}

1. Wolfe, F. et al. The American College of Rheumatology 1990 Criteria for the Classification of Fibromyalgia. Report of the Multicenter Criteria Committee. Arthritis Rheum. 33, 160-172 (1990).

2. Wolfe, F. et al. Fibromyalgia criteria and severity scales for clinical and epidemiological studies: a modification of the ACR Preliminary Diagnostic Criteria for Fibromyalgia. J. Rheumatol. 38, 1113-1122 (2011).

3. Goebel, A., Stock, M., Deacon, R., Sprotte, G. \& Vincent, A. Intravenous immunoglobulin response and evidence for pathogenic antibodies in a case of complex regional pain syndrome 1. Ann. Neurol. 57, 463-464 (2005).

4. Randall, L. O. \& Selitto, J. J. A method for measurement of analgesic activity on inflamed tissue. Arch Int Pharmacodyn Ther 111, 409-419 (1957).

5. Chaplan, S. R., Bach, F. W., Pogrel, J. W., Chung, J. M. \& Yaksh, T. L. Quantitative assessment of tactile allodynia in the rat paw. J. Neurosci. Methods 53, 55-63 (1994).

6. Gentry, C., Stoakley, N., Andersson, D. A. \& Bevan, S. The roles of iPLA2, TRPM8 and TRPA1 in chemically induced cold hypersensitivity. Mol Pain 6, 4 (2010).

7. Andersson, D. A., Gentry, C., Moss, S. \& Bevan, S. Clioquinol and pyrithione activate TRPA1 by increasing intracellular Zn2+. Proc. Natl. Acad. Sci. U.S.A. 106, 8374-8379 (2009).

8. Zimmermann, K. et al. Phenotyping sensory nerve endings in vitro in the mouse. Nat Protoc 4, 174-196 (2009).

9. Vastani, N. et al. Impaired Nociception in the Diabetic Ins2+/Akita Mouse. Diabetes 67, 1650-1662 (2018). 